\title{
SCIENTIFIC SUBSTANTIATION OF EFFICIENCY OF CONTROL OF PHYSICAL PREPATIVITY OF HANDBALL
}

\author{
${ }^{1}$ Zhunisbek D.N.; ${ }^{2}$ Mavrudieva N.; ${ }^{2}$ Kudashova L.R.; ${ }^{2}$ \\ Kefer N. E.; ${ }^{2}$ Andrewishkin I.L., ${ }^{2}$ Kudashov E.S. \\ ${ }^{1}$ National Sports Academy. Vassil Levski, country Bulgaria city Sofia \\ ${ }^{2}$ National Sports Academy. Vassil Levski, country Bulgaria City of Sofia \\ ${ }^{2}$ Kazakh Academy of Sport of Tourism, the country Kazakhstan, Almaty
}

\begin{abstract}
Summary
The urgency of the problem is connected with the increase in the role of universalization of players in the modern handball and their functional specialization, which requires the development of models for their control, evaluation and management of the level of athletic preparedness at each age, training and competitive stage of the training process. The purpose of our research is to increase the effectiveness and reliability of the tests used to comprehensively assess the level of preparedness and selection of more promising female students for employment in handball.

The study was conducted on 80 handball players of various qualifications for creating model characteristics of tests, assessing their significance for controlling physical fitness and influencing competitive activity.

The results of the study made it possible to draw conclusions that the leading motor abilities of handball players, mainly influencing the effectiveness of gaming activity, are: the level of manifestation of speed-power abilities when performing exercises approximate in structure to the specifics of the performance of game techniques; coordination abilities, expressed in the effective performance of game tasks in the variation conflict situations of the game; the ability of athletes to accurately differentiate patio-temporal parameters of motion; the ability to carry out a specific activity for a long time without reducing its effectiveness.

The most significant indicators reflecting the level of performance of the leading motor qualities are: speed of running on short stretches; running speed with the ball; length and height of the jump; power and maximum rate of ballistic movements; accuracy of differentiation of effort; level of general physical working capacity.
\end{abstract}

Key words: student handball, leading physical qualities, selection of effective tests.

Introduction. The rapid growth of sports achievements and the related intensification of the training process, the reduction in the age of champions and record-holders, as well as the early specialization in the opinion of (Bril, 1980), (Guzhalovsky, 1986), (Zatsiorsky, 1979), (Ignatieva et all, 2005) put before the trainers and specialists working with young athletes the problem of selecting able students for various sports activities. The preparation of a perspective reserve in handball is largely hampered by the lack of reasonably well-founded and methodologically developed approach to the process of selecting informative control tests for the level of physical preparedness.

In modern handball at every stage of the training and competitive training processes, the role of functional specialization and universalization of players is increasing, which requires the development of models for managing selection and the level of athletic preparedness by monitoring and evaluating the states of the organism's preparedness in each period of preparation.

Relevance of the research topic. Especially actual is the problem of selecting informative and meaningful tests for control of physical readiness when training players in handball at the university, which is difficult in many respects because of insufficiently substantiated and methodologically developed approaches for the selection process of students who have not previously engaged in this sport. The practice of selection in higher educational institutions is based in the basic on the intuition of trainers-teachers and the application of tests for selection, which do not allow to comprehensively evaluate the perspectives of those engaged. All of the above stated was the subject of this study for the qualitative selection of handball players in the university.

The purpose of these studies is to assess the significance of the indicators being studied for compiling a comprehensive assessment of physical abilities in the formation of teams of handball players of different sports qualifications.

Development and refinement of the most significant indicators for a comprehensive assessment of the level of physical fitness and abilities of female students for playing handball, selecting them for the team of the university, the handball club based on the selection of more informative and reliable control tests.

To achieve this goal, the following research objectives were identified: 
1. Identify the group of the most significant indicators influencing the level of special physical, technical and tactical preparedness in order to select students of sports qualifications in teams.

2. Compare the indices of variation of the tested parameters of physical readiness for handballs - students of different qualifications, for assessing the homogeneity of the selection by physical qualities.

3. Conduct selection of female students in the handball team according to the most informative tests of physical fitness.

To solve the set goals and objectives, we used scientific methods:

1. Analysis of scientific and methodological literature

2. Teaching observations

3. Pedagogical experiment using the following methods: chronometry, chronodinamometry, pedagogical testing, pedagogical observation

4. Method of expert evaluation

5. The method of mathematical statistics.

The study was conducted with the participation of 80 high-qualified athletes, among them MS-12, CCM-24, sportsmen of the 1st category - 24 and 2 categories -20 people. In total, 80 people took part in the study, who did not have any abnormalities in their health status. In order to obtain informational material about the gambling activities of the handball players, the analysis of which helps to more clearly and specifically correct the training of players, numerous pedagogical observations of the gaming activities of members of the leading Kazakhstan handball players and the strongest women's clubs of Kazakhstan were conducted in the present work.
To obtain quantitative indicators, the level of development of the qualities of speed, strength, dexterity, endurance, flexibility, as well as coordination abilities and functions of the state of the organism of sportswomen in handball players in dig research, special pedagogical tests were applied that best reflect the level of development of motor cachets' and show the level of mastery of specific techniques, characteristic for the game of handball.

Long-term research on the preparation of Russian teams in handball shows that the indicators of the integrated test correlate with the effectiveness of the gaming activity of women's handball players. The results of the analysis of scientific work A. Blokhin. (Blokhin, 2003) confirmed the prognostic significance of the "Complex Exercise" test, according to which one can judge the players' preparedness for the forthcoming competitive struggle. The results of this test show a significant decrease in the performance of players by the end of the competition peri$\mathrm{od}$. The remaining tests at the level of higher sportsmanship can give only a general idea of the level of development of physical qualities in handball.

In our studies, we used a complex test, as a generalized universal indicator of the sports skills of the handball players.

In the first series of tests, control tests were tested on high-qualified handball players with the goal of revealing the average level of physical fitness and homogeneity of indicators for high-skilled handball players. The results of testing the physical fitness of handball players of high qualification are presented in Table 1.

Table 1 - Results of average indicators of testing physical fitness of handball players of high qualification

\begin{tabular}{|l|l|l|l|l|}
\hline No & Name of the methodology, test or indicator & $\tilde{O}$ & S & $\begin{array}{l}\text { S\% variation of standard } \\
\text { deviation }\end{array}$ \\
\hline 1. & Complex test, with & 34,6 & 5,79 & 16,7 \\
\hline 2. & Running 30 m, with & 4,9 & 0,41 & 8,4 \\
\hline 3. & Keeping the ball 30 m, with & 5,3 & 0,54 & 10,2 \\
\hline 4. & Throws of the ball (1kg.) For range, m & 19,5 & 3,14 & 16,1 \\
\hline 5. & Transfer of the ball in 30 seconds, the number of times & 20,3 & 2,34 & 14 \\
\hline 6. & The magnitude of the maximum force of the brush, kg & 37,8 & 8,61 & 22,8 \\
\hline 7. & An error occurred while playing back 50\% of the max. force, kg & 2,9 & 0,98 & 33,7 \\
\hline 8 & Long jump from the place, cm & 206,5 & 8,79 & 4,2 \\
\hline 9. & Harvard Spree Test Index, average & 88,5 & 6,54 & 7,4 \\
\hline 10 & Height of jumping up, cm & 49,3 & 5,67 & 11,5 \\
\hline 11. & The burden of a simple motor reaction, with & 0,31 & 0,05 & 16,1 \\
\hline 12. & The test «Boomerang», with & 14,2 & 0,67 & 4,7 \\
\hline & X & & & 13,8 \\
\hline
\end{tabular}


The data given indicate that the coefficient of variation in motor qualities was on the average within $13.8 \%$, which indicates the homogeneity of the group.
In the second series of studies, the correlation between the indicators of the complex test and the level of sports qualification of female students handballers was determined (Table 2).

Table 2 - Correlation dependence of the complex test indicators on the level of sports skills of handball students

\begin{tabular}{|c|l|c|c|c|}
\hline \multirow{2}{*}{ № } & \multirow{2}{*}{ Comparative indicators Coefficient of correlation } & \multicolumn{3}{|c|}{ Coefficient correlation } \\
\cline { 3 - 5 } & & 1 & 2 & 3 \\
\cline { 3 - 5 } & & - & 0,861 & 0,817 \\
\hline 1. & Rating & - & - & 0,724 \\
\hline 2. & The level of sporting achievements & - & - \\
\hline 3. & Comprehensive test & - & - \\
\hline
\end{tabular}

From these 12 indicators, by comparison with the use of the method of rank correlation between the data of the complex test, the leading parameters reflecting the level of manifestation of the basic abilities of the subjects were identified.

To determine whether a complex test is associated with the level of sports handball skill and with the effectiveness of their gaming activities in the second series of studies, a correlation analysis was conducted. To solve this problem, the subjects were ranked according to three indicators: rating (according to the method of expert assessments), the result of the complex test and the level of sports achievements. So, the members of the RK team got 1 place, members of the youth team, RK -2 , club teams -3 .

Sufficiently high correlation values allow us to state that the levels of complex test indicators were higher for masters of sports and lower for handball play- ers of club teams, which gives the right to say that a complex test really reflects the level of game skill of the subjects.

Further, in the third series of studies, calculations of the correlation dependence of each of the tested indicators and the complex pedagogical test were carried out to identify the leading factors that were determined by comparing the test scores with the complex test.

The results of the correlation analysis are presented in Table 3. Thus, the coefficient of correlation of the 30 meters and 30 meters of running with the ball in relation to the complex test of highly skilled handballers is 0.865 , respectively; 0.773 ; jump in length from the place $-0,743$ throw $1 \mathrm{~kg}$. the ball with three steps 0.547 ; error with force differentiation $50 \%$ of maximum 0.497 ; Number of transfers in 30 seconds. 0.652; IGST - 0,627.

Table 3 - Correlation dependence of each of the tested indicators and the complex pedagogical test in handball players of the 2 nd category

\begin{tabular}{|l|c|}
\hline The name of the methodology, test or indicator & $\mathrm{r}$ \\
\hline The «Boomerang» test, with & 0,387 \\
\hline Height of jumping up, cm & 0,418 \\
\hline The burden of a simple motor reaction, with & 0,426 \\
\hline The maximum force of the brush, kg & 0,443 \\
\hline An error occurred while playing back 50\% of the max. force, kg & 0,497 \\
\hline Ball throw (1kg.) For range, m & 0,547 \\
\hline Harvard Special Test Index, units & 0,627 \\
\hline Transfer of the ball to 30 «, the number of times & 0,652 \\
\hline Long jump from the place, cm & 0,743 \\
\hline Keeping the ball 30 m, with & 0,773 \\
\hline Running 30m., With & 0,865 \\
\hline
\end{tabular}


Thus, as the most significant tests evaluated by the correlation coefficient, we selected the following seven tests: run $30 \mathrm{~m}$, keeping the ball $30 \mathrm{~m}$, throw $1 \mathrm{~kg}$. ball on the range, the number of gears for $30 \mathrm{~s}$, the accuracy of $50 \%$ of the maximum effort, IGST, long jump from the place.

In the 4 series of studies, tests were conducted on 8 leading tests, including a complex test, on the pear of the tested female students having the II sports discharge. The obtained data of average quantities of physical readiness and indices of variation, tested parameters for handballers - students of different qualifications are presented in Table 4, which were compared by calculating $t$ criterion of reliability of difference using the Student's table.

We can assume that those indicators that show a significant increase in physical fitness with an increase in the sports qualification of handball players are the most suitable as indicators of selection of students who have not previously engaged in handball. If any qualities significantly affect the effectiveness of gaming activity and in the process of sporting ontogenesis, the player does not change much, they are related to the division of important qualities in a significant extent genetically determined.

Table 4 - Average values of physical readiness and indices of variation, tested parameters for handballers - students of various qualifications

\begin{tabular}{|c|c|c|c|c|c|c|c|c|}
\hline \multirow{4}{*}{ Name of the test } & \multicolumn{8}{|c|}{ Indicators by groups } \\
\hline & \multirow{2}{*}{\multicolumn{2}{|c|}{$\begin{array}{l}\text { MCMC, MS } \\
(\mathrm{n}-15) \\
1 \\
\end{array}$}} & \multirow{2}{*}{\multicolumn{3}{|c|}{$\begin{array}{c}\text { CCM, I category } \\
(\mathrm{n}-48) \\
2\end{array}$}} & \multicolumn{3}{|c|}{ II bit, without bit $(\mathrm{n}-20)$} \\
\hline & & & & & & & & 3 \\
\hline & $\overline{\tilde{O}}$ & $\sigma$ & $\overline{\tilde{O}}$ & $\sigma$ & $\begin{array}{l}\% \text { differences } \\
\text { between } 1 \text { and } \\
2 \text { group }\end{array}$ & $\overline{\tilde{O}}$ & $\sigma$ & $\begin{array}{l}\% \text { difference } \\
\text { between } \\
1 \text { and } \\
3 \text { group } \\
\end{array}$ \\
\hline Running $30 \mathrm{~m}$, with & 4,7 & 0,24 & 5,1 & 0,38 & $-8,5$ & 5,2 & 0,56 & $-10,6$ \\
\hline Keeping the ball $30 \mathrm{~m}, \mathrm{~s}$ & 5,1 & 0,22 & 4,4 & 0,23 & 13,7 & 5,8 & 0,31 & $-13,7$ \\
\hline Comprehensive testing, with & 32,7 & 1,74 & 35,3 & 2,11 & $-7,95$ & 36,9 & 1,91 & $-12,8$ \\
\hline Throw I kg. ball, m & 20,5 & 2,16 & 18,7 & 1,26 & $-9,8$ & 16,3 & 2,24 & $-20,05$ \\
\hline $\begin{array}{l}\text { Number of ball transfers in } 30 \\
\text { c, }\end{array}$ & 21,4 & 1,07 & 11,2 & 1,26 & $-52,3$ & 18,7 & 1,17 & $-12,7$ \\
\hline $\begin{array}{l}\text { An error occurred while } \\
\text { playing back } 50 \% \text { of the max. } \\
\text { force, } \mathrm{kg}\end{array}$ & 2,2 & 1,84 & 3,8 & 2,93 & $-72,7$ & 4,7 & 2,62 & $-113,6$ \\
\hline IGST, standard units. & 90,5 & 9,01 & 86,9 & 11,77 & $-13,16$ & 80,9 & 10,17 & $-10,7$ \\
\hline $\begin{array}{l}\text { The result of the long jump } \\
\text { from the place, } \mathrm{cm}\end{array}$ & 214,2 & 10,05 & 206,4 & 9,69 & $-4,0$ & 200,8 & 8,87 & $-6,3$ \\
\hline
\end{tabular}

If the effectiveness of gaming activity also reveals a positive dynamics accompanying the growth of skill, it means that it refers to the section of necessary and in a large measure of the qualities being formed.

It is to the latter form that we include the indicators obtained using tests with a high differentiated ability. Consequently, these tests are more suitable for selection of students to engage in handball.

An analysis of the percentage of differences between the qualification groups showed a decrease in the test results depending on the level of sports qualification by $4.0-113.6 \%$. Moreover, the lowest result was revealed by the indicator of management by force.

\section{Conclusions:}

1. Leading motor abilities of handballers, mainly influencing the effectiveness of gaming activity are: the level of manifestation of speed-strength abilities when performing exercises approximate in structure to the specifics of the performance of game techniques; ability of athletes to precise differentiation of space-time parameters of motion; the ability to carry out a specific activity for a long time without reducing its effectiveness.

2. The most informative tests for the diagnosis of 
the motor abilities of handball players who have not previously engaged in handball, according to the degree of correlation with the complex test are: - running $30 \mathrm{~m}$. (0.865)

- running $30 \mathrm{~m}$. with the control of the ball (0.773)

- the number of gears in $30 \mathrm{~s}(0.743)$

- long jump from the seat $(0,650)$

- Harvard step test (0.627)

- throwing a ball weighing $1 \mathrm{~kg}$. to the range (0.547)

- the accuracy of reproduction from the maximum force $(0.497)$

The most significant indicators reflecting the level of performance of the leading motor abilities are the following control tests, which should be used for selection of handball players in the team:

- running speed for short distances;

- speed of running with the ball;

- length and height of the jump;

- power and maximum rate of ballistic movements;

- working capacity;

- accuracy of differentiation of effort.
Comprehensive pedagogical test, including sequential execution of game techniques in combination with rapid movements closely correlate with the indicators of technical and tactical mastery of handball players: player rating (0.817) and level of controversial achievements $(0.724)$

\section{References:}

Blokhin, A.V., (2003) Special preparedness of handball players of high qualification in a long competitive period. Candidate of pedagogy - Sciences Moscow -C16

Bril M.S. (1980) Selection in sports games.- Moscow: Physical training and sports. $-127 \mathrm{p}$.

Guzhalovsky A.A. (1986) Textbook for institutes of physical culture / Ed. A. A. Guzhalovsky.- Moscow: Physical training and sports. $-352 \mathrm{p}$.

Ignatiev V. Ya., Portnov Yu.M. (1996) Handball: A textbook for sports universities. - M: BACKGROUND.

Ignatieva V. Ya., Tkhorev VI, Petracheva I. V.(2005) Preparation of handball players at the stage of higher sportsmanship. training. allowance. M .: Physical Culture.

Zatsiorsky V.M. (1979) Basics of sports metrology Text. / V.M. Zatsiorsky. Moscow: Physical training and sports. $-149 \mathrm{p}$. 